Vibrio cholerae motility in aquatic and mucus-mimicking environments 


\title{
Vibrio cholerae motility in aquatic and mucus-mimicking environments
}

\author{
Marianne Grognot, Anisha Mittal, Mattia Mah'moud, Katja M. Taute*
}

Rowland Institute at Harvard, 100 Edwin H. Land Blvd, Cambridge, MA 02142, USA

*corresponding author: taute@rowland.harvard.edu

Cholera disease is caused by Vibrio cholerae infecting the lining of the small intestine and results in severe diarrhea. $V$. cholerae's swimming motility is known to play a crucial role in pathogenicity and may aid the bacteria in crossing the intestinal mucus barrier to reach sites of infection, but the exact mechanisms are unknown. The cell can be either pushed or pulled by its single polar flagellum, but there is no consensus on the resulting repertoire of motility behaviors.

We use high-throughput 3D bacterial tracking to observe $V$. cholerae swimming in buffer, in viscous solutions of the synthetic polymer PVP, and in mucin solutions that may mimic the host environment. We perform a statistical characterization of its motility behavior on the basis of large 3D trajectory datasets. We find that $V$. cholerae performs asymmetric run-reverse-flick motility, consisting of a sequence of a forward run, reversal, and a shorter backward run, followed by a turn by approximately $90^{\circ}$, called a flick, preceding the next forward run. Unlike many run-reverse-flick swimmers, $V$. cholerae's backward runs are much shorter than its forward runs, resulting in an increased effective diffusivity. We also find that the swimming 
43 speed is not constant, but subject to frequent decreases. The turning frequency in mucin

44 matches that observed in buffer. Run-reverse-flick motility and speed fluctuations are present

45 in all environments studied, suggesting that these behaviors may also occur in natural aquatic

46 habitats as well as the host environment.

47

48

49 Importance

50 Cholera disease produces vomiting and severe diarrhea and causes approximately 100,000

51 deaths per year worldwide. The disease is caused by the bacterium Vibrio cholerae colonizing

52 the lining of the small intestine. V. cholerae's ability to swim is known to increase its infectivity,

53 but the underlying mechanisms are not known. One possibility is that swimming may aid in

54 crossing the protective mucus barrier that covers the lining of the small intestine. Our work

55 characterizing how $V$. cholerae swims in environments that mimic properties of the host

56 environment may advance the understanding of how motility contributes to infection.

57

58 


\section{Introduction}

Motility has been recognized as a major virulence factor in $V$. cholerae $(1,2)$, the causative agent of cholera disease, which produces severe watery diarrhea. The $V$. cholerae population recovered from the so-called "rice-water" stool of infected patients shows both stronger motility and greater infectivity than the same strain grown in the lab(3), and non-motile $V$. cholerae mutants show decreased infectivity(4-6). The flagella that drive motility are a key target of the host immune response: flagellins trigger inflammatory responses from the host(7), and immune infant mice carry antibodies that specifically inhibit flagellum-mediated motility(8). Motility has been suggested to aid the bacteria in crossing the intestinal mucus barrier to access sites of colonization(4, 6, 9).

72 Despite the impact of motility on pathogenicity, characterizations of $V$. cholerae's motility 73 behavior have largely been qualitative, and it is commonly described as having the appearance of "shooting stars" in darkfield microscopy(10-12). Its single polar flagellum allows the cell to swim forward or backward, being pushed or pulled by the flagellum, respectively, depending on the direction of flagellar rotation. The shorter backward swimming segments have been reported to be accompanied by random reorientation $(2,13)$. This description is reminiscent of the well-studied "run-tumble" motility of Escherichia coli, where swimming ("running") is driven by counterclockwise (CCW) rotation of the bundled flagella, while intermittent clockwise (CW) rotation by one or more flagella breaks up the bundle and results in short reorientation events

81 called "tumbles". In line with this, expected motility phenotypes of chemotaxis gene deletion 
82 mutants in $V$. cholerae have often been assigned by analogy to $E$. coli(2, 13, 14). More recent work $(15,16)$ has described $V$. cholerae's motility as the "run-reverse-flick" behavior reported

84 for other polarly flagellated Vibrio species(17-19), where the transition from pushing to pulling

85 is accompanied by a reversal, while the opposite transition is accompanied by a turn of approximately $90^{\circ}$ called a "flick". These two views seem superficially similar, but differ in their

87 prediction for how chemotaxis, that is, the bacterium's ability to bias its direction of motion 88 relative to chemical gradients, can be achieved. Neither view has been supported by a full quantitative characterization of $V$. cholerae's motility behavior.

91 Such a characterization, however, is crucial to understanding how motility and chemotaxis

92 contribute to pathogenicity. The role of chemotaxis in pathogenicity is still debated(1). Butler and Camilli $(2,13)$ demonstrated that the infectivity of chemotaxis mutants depends on their motility phenotype, underscoring the need for an accurate, quantitative assessment of such phenotypes. Currently such an assessment is lacking even for wildtype strains.

97 Here we use high-throughput 3D tracking to demonstrate that $V$. cholerae performs run98 reverse-flick motility with variable swimming speed in aquatic environments and quantify the associated behavioral parameters. We show that its motility strategy of asymmetric forward and backward run durations enhances diffusive spreading. To determine whether $V$. cholerae motility behavior differs in environments that mimic physical properties of the host

102 environment, we also observe $V$. cholerae motility in viscous polymer solutions. We show that 
103 this behavior is retained in environments that approximate the physical complexity of natural

104 habitats.

\section{Results}

\section{Vibrio cholera performs run-reverse-flick motility in aquatic environments}

We utilize a high-throughput 3D bacterial tracking technique(20) to gather large datasets of 3D

117 Visual inspection of individual 3D trajectories reveals approximately straight runs, bordered by 118 turns that alternate in magnitude, indicative of run-reverse-flick motility (Fig. 1c). We employ 119 an automated procedure to detect turning events (Methods). Similar to previous reports for run-reverse-flick motility in Vibrio alginolyticus(20) and Caulobacter crescentus(22), the turning

121 angle distribution (Fig. 1d) shows two distinct peaks, one narrow peak near $180^{\circ}$, reflecting

122 reversals, and a second broader peak with a center near $90^{\circ}$, which we attribute to flicks.

123 Flicks are thought to be caused by a buckling instability of the flagellar hook that connects the 124 flagellar filament to the flagellar motor(18). During backward swimming, the flagellum pulls the 
125 cell, and the hook is likely stretched out. When the cell switches to forward swimming, the hook

126 is compressed by the pushing flagellum and can buckle under the load if a critical force or

127 torque threshold is reached. The buckling typically occurs within approximately 10 ms after

128 motor reversal(18). This delay between reversal and buckling-induced reorientation is not

129 resolved in our video rate recordings, thus the observed flick encompasses both events. If no

130 buckling occurs, only a reversal is observed. Thus, multiple reversals may occur in sequence.

131 Because flicks only occur during the transition from backward to forward swimming, two flicks

132 cannot occur in a row. To test these predictions, we analyze the magnitudes of consecutive

133 turns (Fig. 1f) and find that reversals and flicks typically alternate. A reversal can also be

134 followed by another reversal, but we do not observe consecutive flicks. The observed pattern of

135 turning angle magnitudes is thus consistent with run-reverse-flick motility.

$136 V$. cholerae's flagellar motor is driven by a sodium motive force(23). Decreasing the sodium

137 motive force by decreasing the sodium concentration, [ $\mathrm{Na}+]$, at constant ionic strength, results

138 in a lower swimming speed (Supplementary Figure 1f). The concomitant decrease in the

139 occurrence of flicks (Fig. 2) is consistent with the motor torque driving the underlying buckling

140 transition(18). We thus conclude that Vibrio cholerae performs run-reverse-flick motility.

142 Forward runs are longer than backward runs

143 Identifying turn events as flicks and reversals by their magnitude enables us to assign a bacterial

144 orientation to runs without the need to visualize the flagellum. Of the 18,533 complete runs in

145 our dataset, we can identify 4,581 as forward and 8,180 as backward (Methods). Forward runs

146 show right-handed trajectory curvature when swimming along the bottom surface of the 
147 sample chamber, consistent with a left-handed flagellum pushing the cell by CCW rotation(24)

148 (Supplementary Figure 2). We obtain similar average swimming speeds for forward and 149 backward runs of $90 \mu \mathrm{m} / \mathrm{s}$ and $86 \mu \mathrm{m} / \mathrm{s}$, respectively. Run duration distributions for both 150 directions show a peak at approximately $0.08 \mathrm{~s}$, followed by an approximately exponential 151 decay which is rapid for backward runs and slow for forward runs (Fig. 3b). Peaked run duration 152 distributions have also been found for other polarly flagellated bacteria, Vibrio alginolyticus(17) 153 and Caulobacter crescentus(25). On average, forward runs are approximately 3.6 times longer 154 than backward runs. While we observe no correlation in the duration of consecutive forward and backward runs (Fig. 3c), the differences in the duration of forward runs and their 156 subsequent backward runs are exponentially distributed (Fig. 3d), with different time scales 157 dependent on which run type is longer. One key feature of run-reverse-flick motility is that 158 backward runs roughly retrace the preceding forward run. Given similar forward and backward 159 run speeds, the net displacement caused by one such pair of runs is thus determined by their 160 difference in durations. Exponentially distributed run duration differences have also been 161 observed for $V$. alginolyticus(17) and thus may represent a common feature of species 162 performing run-reverse-flick motility.

163 Run durations are expected to affect random spreading of cells(26) (Fig. 3e). The run-reverse164 flick swimmers V. alginolyticus and C. crescentus show similar durations of forward and 165 backward runs(17, 22). Theoretical work allows a prediction of the effective diffusion 166 coefficient characterizing random spreading at long time scales(26). $V$. cholerae's average run 167 duration is similar to that of $V$. alginolyticus, but its asymmetry in forward and backward run 168 durations is expected to yield an $85 \%$ increase in the diffusion coefficient, compared to a 
symmetric scenario with the same average run duration (Fig. 3e, Supplementary Note 1). The

diffusion coefficient is also enhanced by $19 \%$ compared to a scenario where both run durations

171 equal the longer, forward run duration. We thus conclude that $V$. cholerae's asymmetric run

172 durations enhance random spreading of cells.

\section{Run speed modulation}

175 We observe that the swimming speed during run segments is not constant, but subject to 176 substantial, temporary decreases that are readily apparent by visual inspection of trajectories

177 (Fig. 4a). During these events, the bacterium maintains its previous swimming direction (Fig. 4b)

178 but follows it at a decreased speed. We employ an automated detection procedure that

179 identifies these deceleration events as decreases in speed below a threshold that last for at least two consecutive frames. The speed threshold is set at a fixed fraction $\beta$ of a run-specific baseline value (Methods), and we only consider runs with a minimum duration of 10 frames (0.33 s) to ensure that a baseline speed value can be confidently assigned. Although we consider runs of either orientation in the analysis, the runs that meet the duration threshold are likely primarily forward runs. At the selected value of $\beta=0.75$, we obtain a deceleration event frequency of $0.53 \mathrm{~Hz}$ during runs (Methods). While their frequency is similar to the turning frequency, we detect no strong correlation between estimates of the deceleration

187 frequency and the turning frequency obtained for individual bacteria (Supplementary Figure 188 4f). The durations of the deceleration events are exponentially distributed (Fig. 4c), with an 
with a fixed rate of occurrence. The intervals between decelerations, however, show a peaked

distribution with a maximum at $0.12-0.15 \mathrm{~s}$, followed by an exponential tail (Fig. 4d).

To determine whether these drops in speed represent discrete events or a continuous variation

in speed, we examine the dependence of their properties on the event detection threshold.

Their frequency, duration, and relative speed distributions vary continuously with the threshold individual variability.

Errors in the bacterial position determination can produce fluctuations in the measured velocity

203 if the magnitude of the errors is comparable to the true displacement between neighboring

204 frames. The errors on the measured speed are thus larger for slower swimming bacteria. To

205 rule out measurement errors as the source of the observed speed fluctuations, we acquire

206 trajectories for another run-reverse-flick swimmer with a lower swimming speed, Caulobacter

207 crescentus, under the same measurement conditions. We find that the relative variation in run

208 speed is larger for $V$. cholerae than for $C$. crescentus (Supplementary Figure $4 \mathrm{~g}$ ) and thus rule

209 out measurement errors as the cause of the speed variations observed in V. cholerae. We also

210 confirm that deceleration events are not caused by our trajectory filtering method

211 (Supplementary Figure 4a, b). 


\section{Run-reverse-flick motility in mucin solutions}

214 Run-reverse-flick motility has been viewed as an adaptation to marine habitats(27), raising the

215 question of whether this behavior is preserved in environments as physically complex as the

216 host environment where the bacteria have to cross a viscous mucus barrier that protects the

217 intestinal epithelium to reach sites of infection.

218 The primary component of mucus are mucins, large glycoproteins which form a hydrogel with an estimated physiological concentration range of 1-5\%(28). We track $V$. cholerae swimming in solutions of $1.2 \%$ mucin purified from human saliva. Run-reverse-flick motility is still readily apparent from the trajectories (Fig. 5a). We analyze 2,327 3D trajectories of at least $1 \mathrm{~s}$ duration and $20 \mu \mathrm{m} / \mathrm{s}$ average speed. The swimming speed has decreased to $57 \mu \mathrm{m} / \mathrm{s}$ (Fig. $5 \mathrm{~b}$ ). combine a reversal and a reorientation occurring in rapid succession, the increased flick angle

228 indicates a decreased reorientation, consistent with increased drag. The fraction of turn events 229 identified as reversals is very similar in the absence and presence of mucin (Fig. 5e). While the 230 fraction of turn events identified as flicks is slightly lower in mucin compared to in buffer, we 231 attribute this difference the increased difficulty of identifying flicks due to their increased 232 magnitude. We thus conclude that the flick probability is likely very similar in the presence of 
234 the range of $88-93 \%$ in the absence and $81-94 \%$ in the presence of mucus, depending on

235 whether the unidentified turn events are dominated by flicks or reversals.

236 The observed turning frequency of $0.49 \mathrm{~Hz}$ in mucin matches that observed in buffer. The

237 backward and forward run durations of $(0.17 \pm 0.01) \mathrm{s}$ (mean $\pm \mathrm{SE}$ ) and $(0.71 \pm 0.04) \mathrm{s}$,

238 respectively, are also close to the values of $(0.174 \pm 0.002) \mathrm{s}$ and $(0.62 \pm 0.01) \mathrm{s}$ observed in

239 buffer. We also still observe temporary decreases in swimming speed during runs (Fig. 5a) but

240 cannot rule out that inhomogeneities in the mucin solution contribute to them. The relative

241 variability in swimming speeds is slightly increased in the presence of mucin, with a coefficient

242 of variation, defined as the ratio of standard deviation and mean, of relative swimming speeds

243 of 0.29 in mucin and 0.25 in buffer (Fig. 5c).

\section{Run-reverse-flick motility is preserved in dense polymer solutions}

246 The gut environment likely also contains regions that are denser than the dilute mucin solutions

247 used here. To determine whether $V$. cholerae motility behavior differs qualitatively in denser

248 environments, we observe $V$. cholerae motility in solutions of the synthetic, large molecular-

249 weight polymer PVP K90 (Figure 6). Run-reverse-flick motility is still apparent even at

250 macroscopic viscosities more than 50 times that of water where the swimming speed has

251 dropped to $11 \mu \mathrm{m} / \mathrm{s}$ (Fig. 6a-c). With increasing polymer concentration, the associated changes

252 in refractive index cause increasing localization errors. Because these errors can cause

253 ambiguities in trajectory interpretation, we refrain from performing a quantitative turning

254 analysis at this point. Variations in swimming speed during runs are still visually apparent (Fig.

255 6a-c) and occur at a similar relative amplitude as in buffer (Fig. 6e). 
In a Newtonian liquid with viscosity $\eta$, the swimming speed would be expected to decay as $1 / \eta$

257 if the torque of the flagellar motor remains constant. The average swimming speed of

V. cholerae, however, decreases more slowly with the macroscopic viscosity of PVP K90

solutions (Fig. 6d). The flagellar motors of E. coli(29) and V. alginolyticus(30) have been shown

to exhibit a complex torque-speed relationship that can be approximated by a constant torque

regime at low speeds and high loads and a linear decrease in torque as a function of rotation

speed beyond a rotation speed threshold called the knee frequency. A linear torque-speed exponent of 0.46 provides a good approximation to the data.

267 We conclude that run-reverse-flick motility as well as speed variations are present in solutions of both synthetic and natural polymers, and thus may also occur in the host environment.

\section{Strain dependence of observations}

272 While we performed our experiments with strain O395-NT(21), a mutant of classical strain

2730395 that carries a deletion of both subunits of the cholera toxin (ctxAB), visual inspection of

274 trajectories obtained for wildtype 0395 reveals no differences in behavior (Supplementary

275 Figure 3). Both run-reverse-flick motility and speed modulation during runs are present in both

276 strains. Automated trajectory analysis reveals similar turning frequencies as well as very similar

277 average backward and forward run durations for strains O395-NT and wildtype O395 (Table 1). 
278 The observed turning frequency of $0.49 \mathrm{~Hz}$ aligns well with the value of approximately $0.6 \mathrm{~Hz}$ previously reported(31) for 0395-N1 ( $\Delta$ ctxA in 0395(21)), but is much higher than the value of $0.14 \mathrm{~Hz}$ previously reported for the El Tor strain C6709-1(13). Together with the finding that smooth-swimming mutants possess greater infectivity(13), this discrepancy raises the intriguing question whether selection on turning frequency may have contributed to the displacement of classical strains by El Tor strains in the current $V$. cholerae pandemic.

\section{Discussion}

During its life cycle, $V$. cholerae encounters a wide range of different environments. Outside the and as biofilms that grow, for instance, on the surface of phytoplankton, zooplankton and other chitinous particles(32). Inside the host, the bacterium encounters the complex 3D environments of the digestive system with a wide range of viscosities, porosities, and inhomogeneities. While our observations for $V$. cholerae swimming in buffer likely translate to planktonic cells in natural aquatic environments such as brackish waters, the host environment is not characterized well enough to determine with certainty how well the polymer solutions used in our experiments approximate it. $V$. cholerae colonizes the small intestine, which is lined

297 by a loose, non-attached mucus layer(33, 34). For the colon, which additionally possesses a 298 thick attached mucus layer with an estimated mucin concentration of approximately 6\%(35), the concentration of the non-attached, loose layer has been estimated to be approximately 
300 four times lower than that of the attached layer(36), so likely similar to the mucin concentration of $1.2 \%$ used in our experiments. We thus expect that our mucin solutions may approximate the concentration of the loose mucus layer of the small intestine, though properties of the salivary mucin MUC5B used here may differ from those of intestinal mucins. While detailed rheological data for the MUC5B concentrations used here is not available, literature values on other mucins and more dilute MUC5B solutions suggest a macroscopic viscosity in the range of 2-15 cP (Supplementary Note 2).

310 frequency and decreased flick probability for $V$. cholerae in solutions of $2 \%$ porcine gastric

311 mucin, prepared from commercially available, rehydrated dried porcine gastric mucin. Given $V$.

312 cholerae's chemotactic attraction to mucus(37), the reported decrease in turning frequency

313 might reflect a transient chemotactic response to the mucin solution. Our experimental

314 approach limits such transient effects by incubating the bacteria in the motility medium with or

315 without mucus for more than $30 \mathrm{~min}$ to allow for adaptation to the new environment. In

316 addition, reports that rehydrated dried mucus does not recapitulate characteristic rheological

317 properties of purified mucus(38) suggests that the properties of rehydrated and purified mucin

318 solutions may not be comparable(39). The flick probability is expected to depend on the torque

319 exerted by the flagellar motor and thus drops with decreasing sodium motive force which

320 coincides with a drop in speed(18) (Fig. 2). While, at increasing viscosity, the swimming speed

321 also decreases, the motor torque is expected to remain constant or increase(30). A decreased 
322 flick probability is therefore physically not expected at higher viscosity. The recently reported

323 drop in flick probability in mucin(19) might result from the increased difficulty in

324 disambiguating flicks from reversals because of the larger flick angles in mucin.

Work on E. coli swimming in PVP K90 solutions indicates that, due to strong shear thinning, the

327 resulting bacterial motility behavior may depend only on macroscopic viscosities, not detailed rheological properties, with the cell body experiencing the solution viscosity and the flagellum the solvent viscosity(40) (Supplementary Note 2). Shear thinning or other non-Newtonian effects may contribute to the slow decrease in swimming speed with macroscopic viscosity we observe in PVP solutions. Additional contributions may arise, however, from the ability of the flagellar motor to remodel itself by increasing the number of torque-generating stators in response to load increases such that different loads result in different torque-speed curves(41, 42). Mucus and mucin also exhibit strong shear thinning $(39,43)$. The relative decrease in swimming speed we observe in our mucin solutions is similar to that observed in PVP solutions with a macroscopic viscosity of approximately $3 \mathrm{cP}$ (Fig. 6d, Supplementary Note 2). The range of PVP viscosities we cover aligns with the 1 - $30 \mathrm{cP}$ range of macroscopic viscosities determined for mucus from the small intestine(44). Elastic effects are not expected to play a role for either PVP or mucin at the concentrations studied here $(40,45)$. More generally, our

342 our observations may translate to a broader range of polymer solutions. 
344 The swimming speed variations we observe might be related to pauses in swimming which have

345 been reported in Pseudomonas putida(46), Pseudomonas aeruginosa(47), and Azospirillum

346 brasilense(48), or to the pauses in flagellar rotation that have been reported in $E$. coli(49),

347 Rhodobacter sphaeroides(50), and P. aeruginosa(51). For $E$. coli, a correlation between the

348 turning and flagellar pausing frequencies was observed both between mutant strains and

349 between individuals of the same strain(52). We detect no correlation between individual

350 turning and deceleration frequency for $V$. cholerae (Supplementary Figure 4f), but the typically

351 small number of events per trajectory in our data is insufficient to rule out a correlation. We

352 also cannot confidently determine whether deceleration events appear only in forward runs or

353 in both run directions as most backward runs are too short to assign a reliable baseline speed

354 against which variations can be detected. We note that, during the events we observe,

355 swimming never appears to stop, but only decreases in speed, indicating that the flagellum

356 continues to rotate but at a lower speed. In E. coli, the speed of flagellar rotation is varied in

357 response to the load on the flagellar motor by changes in the number of torque-producing

358 stators that drive motor rotation $(41,42)$. Active modulation of flagellar rotation speeds by the

359 chemotaxis signaling system has been reported for proton-driven, unidirectional, peritrichous

360 flagella in V. alginolyticus(53), which conditionally expresses these lateral flagella in addition to

361 its polar flagellum, as well as in Rhizobium meliloti(54). Transient changes in swimming speed in

362 response to changes in oxygen availability have been reported for $A$. brasilense(55) whose

363 motility is driven by a single, polar, bidirectional flagellum. Tethering experiments may be able

364 to more closely determine the nature and determinants of the modulation in flagellar rotation

365 speed that underlie the swimming speed variation we observe in $V$. cholerae. 
V. cholerae's asymmetry in run durations increases its ability to spread randomly (Fig. 3e). In addition, backward swimming segments typically display more trajectory curvature in the vicinity of surfaces than forward swimming segments(56-58) (Supplementary Figure 2). Thus, a bias for forward swimming may further increase dispersal in the vicinity of surfaces compared to a symmetric scenario. While the potential adaptive value of the asymmetric run duration

372 scheme has yet to be evaluated, one possibility is that strong random dispersal is selected for at 373 some point in the $V$. cholerae life cycle. Interestingly, previous work has consistently found that 374 smooth-swimming mutants which suppress turning outcompete the wildtype during 375 infection $(4,13,59,60)$. Such mutants are also expected to display a strongly enhanced effective 376 diffusion coefficient. Another possibility is that asymmetric run durations might have a 377 favorable effect on chemotactic ability, though Altindal et al.(27) have argued that symmetric 378 run durations maximize the chemotactic drift velocity in V. alginolyticus. observed in Shewanella putrefaciens(61) and bears some similarities to $E$. coli's run-tumble motility, where CW rotation does not produce locomotion, but reorientation. These two mechanisms, however, differ in how the degree of reorientation can be controlled. In the run-

384 tumble-like scenario, the magnitude of the reorientation can be increased by prolonging 385 backward run segments, or CW rotation intervals, akin to the relationship between tumble 386 duration and tumble angle that has been observed in E. coli(62). In run-reverse-flick motility, by 387 contrast, the magnitude of flick angles is fixed for each individual by the hydrodynamic drag 
acting on it(20) and is thus expected to be independent of the duration of CW rotation intervals. The average amount of reorientation, however, also depends on the flicking probability determined by the flagellar motor torque, which can vary with environmental conditions such as salinity (Fig. 2) or nutrient concentrations(63). These differences are likely to affect both random motility and chemotaxis. For instance, the chemotactic precision of the runreverse-flick bacterium $V$. alginolyticus has been shown to be enhanced by the chemoattractant-dependent modulation of the flicking probability(63) via the swimming speed.

Future work should address $V$. cholerae's chemotactic mechanism. The well-studied chemotactic strategy of $E$. coli consists of extending average CCW rotation intervals and shortening average CW rotation intervals in response to favorable chemotactic sensory input, thus extending runs up the gradient. The CW bias, that is the fraction of time spent on $\mathrm{CW}$ rotation, thus serves as a convenient proxy for the turning frequency which guides chemotaxis. Other species displaying run-reverse-flick motility, V. alginolyticus(64) and C. crescentus(22), as well as the run-reverse swimmer $P$. aeruginosa(47), instead respond to favorable signals by extending the current flagellar rotation interval regardless of direction, and perform chemotaxis by modulating the turning frequency without substantial change in the bias. While $V$. cholerae's swimming pattern is closer to the latter category, its short CW rotation intervals are reminiscent of $E$. coli's short tumbles.

Butler and Camilli(13) termed smooth-swimming chemotaxis mutants CCW-biased and frequently turning mutants CW-biased, based on the well-characterized motility phenotypes of 
410 the homologous mutants in E. coli., and attributed their differences in infectivity to the bias in

411 flagellar rotation direction. The bias was, however, not explicitly characterized. Given that bias

412 and turning frequency have been shown to be decoupled in other run-reverse-flick-performing

413 bacteria, and that bias and turning frequency are expected to have distinct effects on motility

414 and chemotaxis(26), future work should address which of these factors drive the observed

415 differences in infectivity. Insights might be gained, in particular, from infectivity assays on

416 smooth-swimming, CW-rotating mutants. We envision that quantitative characterization of

417 motility behaviors of mutants with infectivity phenotypes may present a key tool towards a

418 mechanistic understanding of how motility and chemotaxis behaviors contribute to $V$. cholerae

419 pathogenicity.

420

421 
Bacterial culturing. Overnight cultures were inoculated from individual V. cholerae (0395NT(21) or O395) colonies, grown on 1.5\% agar LB5 plates streaked from glycerol stock, and grown to saturation in $2 \mathrm{ml}$ LB5 (see Table 2 for media compositions) at $30^{\circ} \mathrm{C}, 250 \mathrm{rpm}$. All

427 media were complemented with $100 \mu \mathrm{g} / \mathrm{ml}$ kanamycin for the 0395-NT strain. Day cultures were inoculated at a dilution of 1:200 (v/v) in M9GM and grown at $30^{\circ} \mathrm{C}$ to an optical density (OD) between 0.350 and 0.400 at $600 \mathrm{~nm}$, unless specified otherwise. While LB5 and TG have previously been used in $V$. cholerae motility studies $(5,13,23,65-68)$, M9 minimal medium with pyruvate (M9GM) yielded higher swimming speeds and lower nonmotile fractions, similar to another recent study(69). We found that an OD range of $0.35-0.4$ yielded average swimming speeds above $90 \mu \mathrm{m} / \mathrm{s}$ and a typical motile fraction above $85 \%$.

Sample preparation. Sample chambers with a height of approximately $300 \mu \mathrm{m}$ were created by using two strips consisting of 3 layers of parafilm as spacers between a microscopy slide and a \#1 coverslip. The chamber was then heated on a hot plate and pressed to seal. For samples with mucin, only 2 layers of parafilm were used to reduce sample volumes. Cells were diluted by 1:100 in motility medium M9MM (or fresh growth medium during protocol optimization tests displayed in SI Figure 1a,c), incubated at room temperature for 45 minutes, unless specified otherwise, to allow adaptation to the motility medium, and then flowed into the

441 chamber. Cell solutions are only pipetted with cut pipet tips to avoid shear damage to the 442 flagella. For samples with polymer solutions, cells were first pelleted by centrifugation at 2,000 443 rcf for 8 minutes, resuspended in the relevant motility medium, diluted 1:200 in the polymer 
444 solution, incubated at room temperature for 40-60 minutes, and flowed into the chamber. The

445 ends of the filled chamber were then sealed with molten valap (a mixture of vaseline, lanolin,

446 and paraffin) and immediately brought to the microscope for data acquisition. For motility in

447 M9MM, three to five such samples were prepared and inspected within a period of 10 min for

448 each of three biological replicates for strain O395-NT and one for wildtype O395. For PVP

449 polymer solution experiment, one sample was prepared and inspected per concentration

450 studied, all within a period of 30 minutes. For growth condition tests and mucin solution

451 experiment, two to three samples were prepared and inspected for one biological replicate.

452 Data acquisition. Phase contrast microscopy recordings were obtained at room temperature

$453\left(\sim 22^{\circ} \mathrm{C}\right)$ on a Nikon Ti-E inverted microscope using an sCMOS camera (PCO Edge 4.2, pixel size

$4546.5 \mu \mathrm{m}$ ) and a 40x objective lens (Nikon CFI SPlan Fluor ELWD 40x ADM Ph2, correction collar

455 set to $1.2 \mathrm{~mm}$ to induce spherical aberrations(20)) focused approximately $130 \mu \mathrm{m}$ above the

456 chamber's internal bottom surface. The illumination was adjusted to yield approximately

45720,000 counts per pixel. Recordings were saved as 16-bit tiff files. For each sample in M9MM,

458 one recording with a duration of 2-2.2 $\mathrm{min}$ and a frame rate of $30 \mathrm{fps}$ was obtained

459 immediately after placing the sample on the microscope. The three replicate experiments for

460 O395-NT generated a cumulative $23 \mathrm{~min}$ of video recordings. For each PVP sample, one

461 recording was obtained at $30 \mathrm{fps}$ or $15 \mathrm{fps}$ for concentrations above or under $3 \%$, respectively.

462 During the same experiment, one control acquisition in TMN at $30 \mathrm{fps}$ was done before, in the

463 middle and after acquisitions in PVP. For mucin samples, two 1.7-minute recordings were

464 obtained at $15 \mathrm{fps}$. For growth media tests, two to three recordings of 1 to 3 minutes were 
obtained at $30 \mathrm{fps}$. Numbers of biological and technical replicates, as well as duration of acquisitions, are summarized in Supplementary Table 1.

Data analysis. Video recordings were binned by a factor of $2 \times 2$ by averaging counts and then subjected to a background correction procedure based on dividing the image by a pixel-wise median computed across a sliding window of 101 frames, except for data acquired for mucin experiments, where a sliding window of 41 frames was used. 3D Trajectories were extracted

471 from phase contrast recordings using a high-throughput 3D tracking method based on image 472 similarity between bacteria and a reference library(20). 3D bacterial trajectories were extracted 473 in a tracking volume of approximately $350 \mu \mathrm{m} \times 300 \mu \mathrm{m}$ laterally $(\mathrm{x}, \mathrm{y})$ and $200 \mu \mathrm{m}$ in depth (z)

474 for typically several dozen individuals at a time. Positions were smoothed using $2^{\text {nd }}$ order 475 ADMM-based trend-filtering(70) with regularization parameter $\lambda=0.3$ unless stated otherwise 476 (see Supplementary Table 1), and three-dimensional velocities computed as forward 477 differences in positions divided by the time interval between frames. All trajectories with an 478 average speed below a $20 \mu \mathrm{m} / \mathrm{s}$ threshold, unless stated otherwise, were deemed non-motile 479 and discarded. For PVP polymer solutions, the non-motile threshold was adjusted to the 480 population's swimming speed (see Supplementary Table 1). For samples with 1.3 and $7.4 \mathrm{mM}$ $481 \mathrm{Na}^{+}$, we used $\lambda=0.8$ and a non-motile threshold of $10 \mu \mathrm{m} / \mathrm{s}$.

482 Run-reverse-flick analysis. The bacteria's motility behavior in M9MM was analyzed based on 483 trajectories with a duration of at least $1 \mathrm{~s}$, totaling 23,062 trajectories $(58,029 \mathrm{~s})$ and 8,017 484 trajectories $(19,113 \mathrm{~s})$ for strain 0395-NT and 0395, respectively. The turning event detection is 485 based on the local rate of angular change, computed from the dot product between the sums 486 of the two consecutive velocity vectors preceding and subsequent to a time point. The 
487 threshold for a turn to begin is an $\alpha$-fold rate relative to the median rate of angular change 488 during the trajectory's run segments, as determined in three iterations of the procedure. We 489 determined by visual inspection of trajectories that a factor $\alpha=8$ gave satisfactory results. A 490 turn ends when the local rate of angular change is below the threshold again. The 3D turning 491 angle for a turn beginning at frame $\mathrm{i}$ and ending at point $\mathrm{j}$ is computed as the angle between 492 the sum of the instantaneous velocity vectors at frames $\mathrm{i}-2$ and $\mathrm{i}-1$ and the sum of those at 493 frames $\mathrm{j}$ and $\mathrm{j}+1$. Turning events were labeled flicks or reversals if the turning angle was below $494140^{\circ}$ or above $150^{\circ}$, respectively. The bacterial orientation during runs (forward/backward) was assigned based on the identity of the two bordering turning events. Backward and forward runs were identified as runs with a flick respectively at the end or at the beginning of the run and a 497 reversal at the other end of the run. For the motility analysis of the 0395-NT strain, a total of 8,180 backward and 4,581 forward runs were identified out of 18,533 runs, within a subpopulation of 5,932 trajectories (20,299 s cumulative duration). For the motility analysis of 500 the wildtype 0395 strain, 2,283 backward and 1,240 forward runs were identified out of 5,425 501 runs, within a subpopulation of 1,765 trajectories with a cumulative duration of 5,816 s. For 502 experiments on 0395-NT in sodium concentrations of $136 \mathrm{mM}, 7.4 \mathrm{mM}$ and $1.8 \mathrm{mM}$, displayed 503 in Figure 2, we detected 2,769, 6,093 and 10,035 turning events with measurable turning 504 angles, respectively, in 2,683, 5,089 and 4,083 trajectories. For 0395 in mucin solutions, we 505 obtained and analyzed 2,327 trajectories with a total duration of 6,027 s, containing 2,587 506 turning events with measurable turning angle, and enabling us to identify 707 backward and 507385 forward runs. 
Deceleration analysis. Runs with a duration of more than $0.33 \mathrm{~s}$ and an average speed above

$30 \mu \mathrm{m} / \mathrm{s}$ were screened for segments of decreased speed, here called "decelerations". A deceleration begins when the instantaneous speed drops below a threshold for two consecutive frames and ends when the speed is above the threshold again for two consecutive

512 frames. The threshold is defined as a fraction $\beta$ of the run's median speed outside of 513 deceleration events, as determined iteratively in two rounds of the detection procedure. We

514 tested a range of $\beta=0.3-0.9$ (Supplementary Figure 4). Figure 4 shows results obtained for $\beta=$ 5150.75.

Polymer solutions. O395-NT was tracked in solutions of the linear polymer polyvinylpyrrolidone

517 (PVP K90, Sigma 81440, average molecular weight of about 360,000 kDa) with concentrations of 518 ranging from 0.9 to $6.7 \%(\mathrm{w} / \mathrm{w})$ in $\mathrm{TMN}$, or of human MUC5B mucin purified from human 519 saliva(71) (a kind gift of $\mathrm{K}$. Ribbeck) at 1.2\% (w/w) in M9MM. $3.3 \mathrm{mg}$ lyophilized mucin was 520 dissolved in $275 \mu \mathrm{M}$ MMM by shaking at $250 \mathrm{rpm}$ at $4^{\circ} \mathrm{C}$ for 5 hours. Two hours before the 521 experiment, the solution was slowly pipetted to further homogenize it, and shaken at room 522 temperature at $300 \mathrm{rpm}$. Macroscopic viscosity measurements for PVP K90 solutions with 523 concentrations in the $0 \%-7.2 \%$ range were obtained using two falling-ball viscometers 524 (Gilmont, $2-20 \mathrm{cP}$ range and $10-100 \mathrm{cP}$ range) at room temperature $\left(21^{\circ} \mathrm{C}\right)$, after calibration 525 using viscosity standards of 11.6 and 48.0 cP (Parangon Scientific, general purpose standards 526 D10 and N26, respectively). The relationship between PVP concentration and viscosity was 527 extracted as a second order polynomial fit with a forced intercept of $0.98 \mathrm{cP}$ at $0 \%$ PVP 528 (Supplementary Figure 5). 
529 Low sodium motility experiment. We varied the motility medium's $\mathrm{Na}^{+}$concentration at

530 constant ionic strength by mixing M9MM with a buffer identical to M9MM except that all

531 sodium-containing ingredients were replaced by the equivalent potassium-containing ones.

$532 \mathrm{NaCl}$ was replaced by $\mathrm{KCl}$, and $\mathrm{Na}_{2} \mathrm{HPO}_{4}$ was replaced by $\mathrm{K}_{2} \mathrm{HPO}_{4}$ (NoNa-M9MM, see Table 2).

533 The experiments were performed as described for experiments in M9MM, except that the

534 culture was not diluted into the motility medium but pelleted at 2,000 rcf for 8 minutes before

535 gentle resuspension in the appropriate motility medium in order to precisely control the final

$536 \mathrm{Na}^{+}$concentration.

537 C. crescentus experiment. C. crescentus has a dimorphic life cycle: a stalked cell attached to a 538 surface divides and releases a motile daughter cell. We obtained motile cells by a modified 539 plate-release protocol(72). An overnight culture inoculated from an individual C. crescentus 540 (CB15, ATCC 19089) colony, grown on 1.5\% agar PYE plates streaked from frozen glycerol stock 541 stored at $-80^{\circ} \mathrm{C}$, was grown to saturation in $2 \mathrm{ml} \mathrm{PYE} \mathrm{at} 30^{\circ} \mathrm{C}, 200 \mathrm{rpm}$. The overnight culture 542 was diluted 1:100 in fresh PYE, then grown in volumes of $0.5 \mathrm{ml}$ in a 24-well plate incubated at $54330^{\circ} \mathrm{C}$ without agitation for 24 hours. The wells were washed and refilled with fresh PYE, placed 544 at room temperature on a shaker at $130 \mathrm{rpm}$ for another 24 hours. At that stage, the bottoms 545 of the wells were covered with a dense carpet of stalked cells, continuously producing motile 546 cells. For experiments, a well was rinsed three times with PYE, then $0.5 \mathrm{ml}$ of fresh PYE were 547 placed in the well and removed after 5 minutes, containing the newly separated motile cells. 548 Such cell suspensions were immediately injected into a sample chamber and placed on the 549 microscope. Recordings with a duration of 1.5 min were obtained as for $V$. cholerae. Data 
bioRxiv preprint doi: https://doi.org/10.1101/2021.07.06.451398; this version posted July 7, 2021. The copyright holder for this preprint (which was not certified by peer review) is the author/funder, who has granted bioRxiv a license to display the preprint in perpetuity. It is made available under aCC-BY-NC-ND 4.0 International license.

analysis was performed as for $V$. cholerae, except a threshold factor $\alpha=6$ was used for turn

551 event detection as it seemed to produce more accurate results based on visual inspection.

552

553

554 
555 Figure 1: $V$. cholerae 3D motility characterization.

556

557

558

559

560

561

562

563

564

565

566

567

568

569

570

571

572 a) 3D trajectories obtained from one $100 \mathrm{~s}$-long video recording.

b) Probability distribution of average individual swimming speeds of the full population (black, weighted by trajectory duration) and of instantaneous swimming speeds for the analyzed population (red), consisting of trajectories with an average speed larger than $20 \mu \mathrm{m} / \mathrm{s}$ (marked by dashed line) and a minimal duration of $1 \mathrm{~s}$.

c) Example trajectory with color reflecting swimming speed. The arrow marks the trajectory start.

d) Distribution of turning angles and classification of turn events. Turns by less than $140^{\circ}$ are considered flicks, and those by more than $150^{\circ}$ are considered reversals. Flick and reversal angles have magnitudes of $88^{\circ} \pm 26^{\circ}$ (mean \pm SD) and $169^{\circ} \pm 6^{\circ}$, respectively.

e) Turn event identification in the trajectory from panel c reveals alternating flicks (orange) and reversals (teal).

f) Bivariate histogram of consecutive turning angles observed after versus before the same run. Reversals can be preceded and followed by reversals or flicks, but two flicks never occur in a row. 
bioRxiv preprint doi: https://doi.org/10.1101/2021.07.06.451398; this version posted July 7, 2021. The copyright holder for this preprint (which was not certified by peer review) is the author/funder, who has granted bioRxiv a license to display the preprint in perpetuity. It is made available under aCC-BY-NC-ND 4.0 International license.

573 Figure 2: V. cholerae flicking probability depends on the sodium motive force. Bivariate

574 histograms of consecutive turning angles for sodium concentrations of $136 \mathrm{mM}(\mathrm{a}), 7.4 \mathrm{mM}$ (b)

575 and $1.8 \mathrm{mM}(\mathrm{c})$, with respective average swimming speeds of 92,37 , and $23 \mu \mathrm{m} / \mathrm{s}$. The sodium

576 concentration for Fig. $1 \mathrm{f}$ is $181 \mathrm{mM}$ and the average swimming speed $94 \mu \mathrm{m} / \mathrm{s}$.

577 
578 Figure 3: Run characterization.

579 a) Example trajectory from Fig. 1c with runs identified as forward (blue) or backward (red) based on the identity of the bordering turning events (orange: flicks, teal: reversals).

b) Run duration distributions. Average durations of backward (red) and forward (blue) runs are $0.174 \pm 0.002 \mathrm{~s}$ (mean $\pm \mathrm{SE}$ ) and $0.62 \pm 0.01 \mathrm{~s}$, respectively. Inset: Fraction of runs that are longer than a threshold, $d$, as a function of $d$. Line fits in log-linear space to the ranges of 0.1-1.5 s and 0.067-0.4 s yield exponential decay time scales of $0.60 \mathrm{~s}$ and 0.14 s for forward and backward runs, respectively. The tail of the backward run duration distribution likely represents misidentified forward runs.

c) Relationship between backward and forward run duration. Purple: Average duration of backward runs as a function of the preceding forward run's duration; black: average duration of backward runs as a function of average duration of forward runs for trajectories containing at least 4 runs of known orientation. subsequent backward run, shown in cyan (magenta) when the forward run is longer (shorter) than the backward. Inset: Fraction of events that are longer than a threshold, $x$, as a function of $x$. Partially transparent lines indicate exponential decay fits. For positive differences (cyan), a maximum likelihood fit of an exponential distribution yields an exponential decay time of $0.60 \mathrm{~s}$. For negative differences (magenta), the slope of a line fit in semi-log space in the range of $0-0.8 \mathrm{~s}$ yields a decay time of $0.14 \mathrm{~s}$. We attribute the tail of the distribution for negative differences to misidentified forward runs, as in panel b. 
600

601

602

603

604

605

606

607

608

609 e) Predicted effective diffusion coefficient for run-reverse-flick motility as a function of the average backward run duration, $\tau_{b}$, based on results of Taktikos et al.(26) (see Supplementary Note 1 for details). The black solid line indicates a fixed forward run duration equal to the measured value, $\tau_{f}=\tau_{f}^{m}=0.62 \mathrm{~s}$, and the black dashed line indicates equal forward and backward run durations. Grey dashed lines mark a scenario where both forward and backward run durations are equal to the average measured run duration. Gray dotted lines mark the scenario where both equal the measured forward run duration. Grey solid lines indicate the measured scenario of $\tau_{f}{ }^{m}=0.62 \mathrm{~s}$ and $\tau_{b}{ }^{m}=$ $0.174 \mathrm{~s}$. 
610 Figure 4: Deceleration events.

611 a) Example trajectory with visually apparent segments of decreased speed marked by arrows.

612 b) Time series of swimming speed (top) and angular change in swimming direction between

613 consecutive frames (bottom) for the trajectory shown in panel a. Blue segments represent

614 forward swimming, red segments backward swimming.

615 c) Distribution of durations of deceleration events. The average duration is $0.12 \mathrm{~s}$. Inset:

616 Fraction of events longer than a threshold, $d$, as a function of $d$. The slope of a linear fit (red) in

617 in semi-log space yields an exponential decay time of $0.094 \mathrm{~s}$.

618 d) Distribution of time between two consecutive decelerations. The average is $0.41 \mathrm{~s}$. Inset:

619 Fraction of events longer than a threshold, $d$, as a function of $d$. The slope of a linear fit (red) in

620 the range of $0.16-2 \mathrm{~s}$ in semi-log space yields an exponential decay time constant of $0.38 \mathrm{~s}$. 
621 Figure 5: Run-reverse-flick motility in solutions of $1.2 \%$ mucin in M9MM.

622 a) Three example trajectories in $1.2 \%$ mucin, with marked reverse, flick, and deceleration 623 events.

624 b) Distribution of instantaneous swimming speeds observed in the presence (red) and absence

625 (blue) of mucin. The average speeds are $57 \mu \mathrm{m} / \mathrm{s}$ in mucin and $94 \mu \mathrm{m} / \mathrm{s}$ in M9MM.

c) Distribution of relative swimming speeds in the presence (red) and absence (blue) of mucin.

627 The relative speed is the instantaneous swimming speed divided by the individual's median 628 swimming speed.

d) Bivariate histogram of consecutive turning angles in $1.2 \%$ mucin also displays alternating

flicks and reversals as well as consecutive reversals, consistent with run-reverse-flick motility.

e) Distribution of turning angles and classification of turn events for trajectories in M9MM

632 (grey, reproduced from Fig. 1d) and in mucin (black). The average flick angle is $88^{\circ} \pm 26^{\circ}$ (mean

$633 \pm \mathrm{SD}$ ) in $\mathrm{M9MM}$ and $97^{\circ} \pm 33^{\circ}$ in $1.2 \%$ mucin/M9MM. The average reversal angle is $169^{\circ} \pm 6^{\circ}$ in

$634 \mathrm{M} 9 \mathrm{MM}$ and $168 \pm 7^{\circ}$ in mucin.

635 f) Fraction of turn events classified as flicks (orange), reversals (teal), or unidentified (gray) in 636 the absence and presence of $1.2 \%$ mucin. Turns of an angle up to $140^{\circ}$ are considered flicks,

637 those above $150^{\circ}$ reversals, and those in between $140^{\circ}$ and $150^{\circ}$ are considered unidentified.

638 Absolute numbers of events for each category are given. 
640 Figure 6: Run-reverse-flick motility in PVP K90 solutions.

641 a-c) Example trajectories showing run-reverse-flick motility and variations in swimming speed at 642 concentrations of $2.2 \%, 4.5 \%$, and 6\% PVP K90 in TMN, respectively.

643 d) Average swimming speed as a function of macroscopic PVP K90 viscosity. The dashed and 644 dotted lines represent two different motor torque models under the assumption of Newtonian 645 fluid behavior. The light gray, dotted line indicates a $\eta^{-1}$ dependence corresponding to a 646 constant motor torque. The darker dashed line is a fit of the dependence $v=a /(\eta+b)$ that is 647 expected for a linear torque-speed relationship(40). Parameters $a=588 \mu \mathrm{m} \mathrm{cP} \mathrm{s}^{-1}$ and $\mathrm{b}=9.7 \mathrm{cP}$ 648 yield the best error-weighted fit when a fixed relative measurement error in swimming speed is 649 assumed. The gray solid line represents a power law fit with a dependence $\eta^{-0.46}$.

650 e) Distribution of relative swimming speeds for different PVP concentrations, reflecting 651 constant variability of swimming speeds. The relative speed is the instantaneous swimming 652 speed divided by the individual's median swimming speed. 


\section{Table 1}

\begin{tabular}{|c|c|c|c|c|c|}
\hline & Analyzed & Average & & Average & Average \\
Strain & trajectory & swimming & Turning & forward run & backward run \\
& time & speed & frequency & duration & duration \\
\hline O395-NT & $58,029 \mathrm{~s}$ & \multirow{2}{*}{$94 \mu \mathrm{m} / \mathrm{s}$} & $0.49 \mathrm{~Hz}$ & $0.62 \pm 0.01 \mathrm{~s}$ & $0.174 \pm 0.002 \mathrm{~s}$ \\
& $(23,062)$ & & $(28,703)$ & $(4,581)$ & $(8,180)$ \\
\hline O395 (wt) & $19,113 \mathrm{~s}$ & \multirow{2}{*}{$8.5 \mu \mathrm{m} / \mathrm{s}$} & $0.47 \mathrm{~Hz}$ & $0.58 \pm 0.02 \mathrm{~s}$ & $0.18 \pm 0.01 \mathrm{~s}$ \\
& $(8,017)$ & & $(8,978)$ & $(1,240)$ & $(2,283)$ \\
\hline
\end{tabular}


bioRxiv preprint doi: https://doi.org/10.1101/2021.07.06.451398; this version posted July 7, 2021. The copyright holder for this preprint (which was not certified by peer review) is the author/funder, who has granted bioRxiv a license to display the preprint in perpetuity. It is made available under aCC-BY-NC-ND 4.0 International license.

\section{Table 2}

\begin{tabular}{|c|c|}
\hline LB5 & $\begin{array}{l}1 \% \text { Bacto Tryptone } \\
0.5 \% \text { Bacto Yeast extract } \\
0.5 \% \mathrm{NaCl} \\
\mathrm{pH} 7.0\end{array}$ \\
\hline LB10 & $\begin{array}{l}\text { 1\% Bacto Tryptone } \\
0.5 \% \text { Bacto Yeast extract } \\
1 \% \mathrm{NaCl} \\
\mathrm{pH} 7.0\end{array}$ \\
\hline TG & $\begin{array}{l}\text { 1\% Bacto Tryptone } \\
0.5 \% \mathrm{NaCl} \\
0.5 \%(\mathrm{w} / \mathrm{v}) \text { Glycerol } \\
\mathrm{pH} 7.1\end{array}$ \\
\hline M9GM & 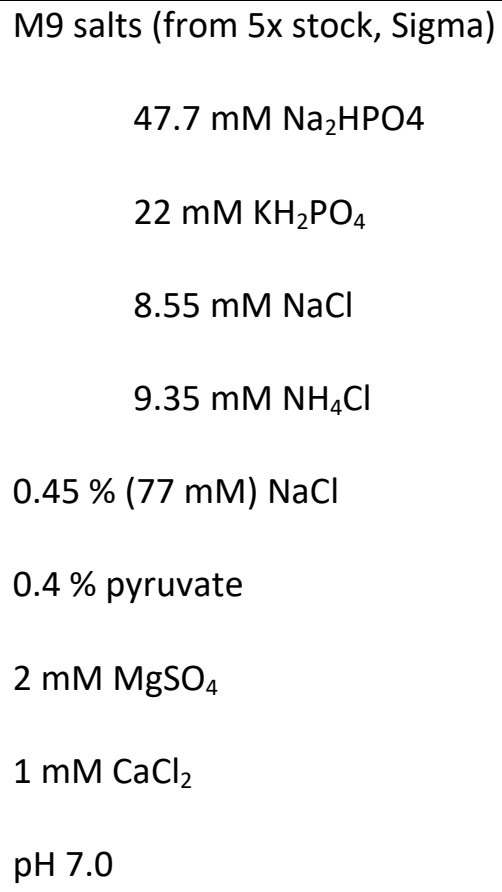 \\
\hline
\end{tabular}




\begin{tabular}{|c|c|}
\hline M9MM & $\begin{array}{l}\text { M9 salts (from } 5 \times \text { stock, Sigma) } \\
47.7 \mathrm{mM} \mathrm{Na}_{2} \mathrm{HPO}_{4} \\
22 \mathrm{mM} \mathrm{KH}_{2} \mathrm{PO}_{4} \\
8.55 \mathrm{mM} \mathrm{NaCl} \\
9.35 \mathrm{mM} \mathrm{NH}_{4} \mathrm{Cl} \\
77 \mathrm{mM} \mathrm{NaCl} \\
5 \mathrm{mM} \mathrm{glucose} \\
2 \mathrm{mM} \mathrm{MgSO}{ }_{4} \\
1 \mathrm{mM} \mathrm{CaCl} \\
\mathrm{pH} 7.0\end{array}$ \\
\hline NoNa-M9MM & $\begin{array}{l}47.7 \mathrm{mM} \mathrm{K}_{2} \mathrm{HPO}_{4} \\
22 \mathrm{mM} \mathrm{KH}_{2} \mathrm{PO}_{4} \\
8.55 \mathrm{mM} \mathrm{KCl} \\
9.35 \mathrm{mM} \mathrm{NH}_{4} \mathrm{Cl} \\
5 \mathrm{mM} \mathrm{glucose}^{2 \mathrm{mM} \mathrm{MgSO}_{4}} \\
1 \mathrm{mM} \mathrm{CaCl} \\
\mathrm{pH} 7.0\end{array}$ \\
\hline TMN & $\begin{array}{l}50 \mathrm{mM} \text { Tris- } \mathrm{HCl} \\
300 \mathrm{mM} \mathrm{NaCl} \\
5 \mathrm{mM} \mathrm{MgCl}_{2} \\
5 \mathrm{mM} \text { glucose } \\
\mathrm{pH} 7.5\end{array}$ \\
\hline PYE & 0.2\% Bacto Peptone \\
\hline
\end{tabular}


bioRxiv preprint doi: https://doi.org/10.1101/2021.07.06.451398; this version posted July 7, 2021. The copyright holder for this preprint (which was not certified by peer review) is the author/funder, who has granted bioRxiv a license to display the preprint in perpetuity. It is made available under aCC-BY-NC-ND 4.0 International license.

\section{9}

Table 2: Composition of growth and motility media.

660

661 


\section{Acknowledgments}

663 Purified mucin was a kind gift from Katharina Ribbeck (MIT). V. cholerae strains 0395 and O395-

664 NT were a kind gift from Edward Ryan (MGH/Harvard Medical School). We thank Chloe M. Wu

665 for advice on mucin experiments. This research was supported by the Rowland Institute as well

666 as the Rowland Institute Postdoctoral Fellow and Undergraduate Program, funded by the

667 Rowland Foundation.

668

669

670

Data availability

671 All data and computer codes are available from the authors upon reasonable request.

672

673

674

\section{Author contributions}

675 K.M.T. and M.G. conceived the research. M.M., A.M., and M.G. performed growth condition

676 tests. A.M. and M.G. performed all other experiments on V. cholera. M.G. performed

677 experiments on $C$. crescentus as well as viscosity measurements. M.G. developed the turning event analysis. A.M. and M.G. contributed to performing and evaluating turning event analysis.

A.M. noticed deceleration events, M.G. developed and performed deceleration event analysis. 


\section{References}

685

686

687 1. Almagro-Moreno S, Pruss K, Taylor RK. 2015. Intestinal Colonization Dynamics of Vibrio

688 cholerae. PLoS Pathog 11:e1004787-11.

689

2. Butler SM, Camilli A. 2005. Going against the grain: chemotaxis and infection in Vibrio cholerae.

690 Nature Reviews Microbiology 3:611-620.

691

3. Butler SM, Nelson EJ, Chowdhury N, Faruque SM, Calderwood SB, Camilli A. 2006. Cholera stool

692 bacteria repress chemotaxis to increase infectivity. Mol Microbiol 60:417-426.

4. Lee SH, Butler SM, Camilli A. 2001. Selection for in vivo regulators of bacterial virulence. Proceedings of the National Academy of Sciences 98:6889-6894.

695

5. Silva AJ, Leitch GJ, Camilli A, Benitez JA. 2006. Contribution of Hemagglutinin/Protease and Motility to the Pathogenesis of El Tor Biotype Cholera. Infect Immun 74:2072-2079.

697 6. Liu Z, Miyashiro T, Tsou A, Hsiao A, Goulian M, Zhu J. 2008. Mucosal penetration primes Vibrio 698 cholerae for host colonization by repressing quorum sensing. Proc Natl Acad Sci U S A 105:97699774.

$7007 . \quad$ Rui H, Ritchie JM, Bronson RT, Mekalanos JJ, Zhang Y, Waldor MK. 2010. Reactogenicity of liveattenuated Vibrio cholerae vaccines is dependent on flagellins. Proceedings of the National Academy of Sciences 107:4359-4364.

703 8. Wang Z, Lazinski DW, Camilli A. 2016. Immunity Provided by an Outer Membrane Vesicle Cholera Vaccine Is Due to O-Antigen-Specific Antibodies Inhibiting Bacterial Motility. Infect Immun 85:209-9. 
9. Millet YA, Alvarez D, Ringgaard S, von Andrian UH, Davis BM, Waldor MK. 2014. Insights into Vibrio cholerae Intestinal Colonization from Monitoring Fluorescently Labeled Bacteria. PLoS Pathog 10:e1004405-14.

10. Benenson AS, Islam MR, Greenough WB. 1964. RAPID IDENTIFICATION OF VIBRIO CHOLERAE BY DARKFIELD MICROSCOPY. Bull World Health Organ 30:827-831.

11. Weil AA, LaRocque RC. 2020. 47 - Cholera and Other Vibrios, Tenth Edition ed doi:10.1016/B9780-323-55512-8.00047-8. Elsevier Inc.

12. Chowdhury F, Khan Al, Faruque ASG, Ryan ET. 2010. Severe, acute watery diarrhea in an adult. PLoS Negl Trop Dis 4:e898.

13. Butler SM, Camilli A. 2004. Both chemotaxis and net motility greatly influence the infectivity of Vibrio cholerae. Proceedings of the National Academy of Sciences 101:5018-5023.

14. Ringgaard S, Schirner K, Davis BM, Waldor MK. 2011. A family of ParA-like ATPases promotes cell pole maturation by facilitating polar localization of chemotaxis proteins. Genes \&amp; Development 25:1544-1555.

15. Ortega DR, Kjaer A, Briegel A. 2020. The chemosensory systems of Vibrio cholerae. Mol Microbiol 199:3289.

16. Ringgaard S, Yang W, Alvarado A, Schirner K, Briegel A, DiRita VJ. 2018. Chemotaxis Arrays in Vibrio Species and Their Intracellular Positioning by the ParC/ParP System. J Bacteriol 200:26717.

17. Xie L, Altindal T, Chattopadhyay S, Wu X-I. 2011. Bacterial flagellum as a propeller and as a rudder for efficient chemotaxis. Proceedings of the National Academy of Sciences 108:22462251.

18. Son K, Guasto JS, Stocker R. 2013. Bacteria can exploit a flagellar buckling instability to change direction. Nature Physics 9:494-498. 
19. Frederick A, Huang Y, Pu M, Rowe-Magnus DA. 2020. Vibrio cholerae Type VI Activity Alters Motility Behavior in Mucin. J Bacteriol 202.

732 20. Taute KM, Gude S, Tans SJ, Shimizu TS. 2015. High-throughput 3D tracking of bacteria on a standard phase contrast microscope. Nature Communications 6:1-9.

21. Mekalanos JJ, Swartz DJ, Pearson GDN, Harford N, Groyne F, de Wilde M. 1983. Cholera toxin genes: nucleotide sequence, deletion analysis and vaccine development. Nature 306:551-557.

22. Grognot M, Taute KM. 2021. A multiscale 3D chemotaxis assay reveals bacterial navigation mechanisms. Communications Biology 4:669.

23. Kojima S, Yamamoto K, Kawagishi I, Homma M. 1999. The polar flagellar motor of Vibrio cholerae is driven by an Na+ motive force. J Bacteriol 181:1927-1930. near Solid Boundaries. Biophys J 90:400-412.

742 25. Morse M, Bell J, Li G, Tang JX. 2015. Flagellar Motor Switching in Caulobacter Crescentus Obeys First Passage Time Statistics. Phys Rev Lett 115:312.

744 26. Taktikos J, Stark H, Zaburdaev V. 2013. How the Motility Pattern of Bacteria Affects Their $745 \quad$ Dispersal and Chemotaxis. PLoS One 8:e81936-16.

746 27. Altindal T, Xie L, Wu X-I. 2011. Implications of Three-Step Swimming Patterns in Bacterial 747 Chemotaxis. Biophysj 100:32-41.

748 28. Bansil R, Turner BS. 2017. The biology of mucus: Composition, synthesis and organization. Advanced drug delivery reviews 124:3-15.

750 29. Chen X, Berg HC. 2000. Torque-Speed Relationship of the Flagellar Rotary Motor of Escherichia coli. Biophys J 78:1036-1041.

752 30. Sowa Y, Hotta H, Homma M, Ishijima A. 2003. Torque-speed Relationship of the Na+-driven 
31. Hyakutake A, Homma M, Austin MJ, Boin MA, Häse CC, Kawagishi I. 2005. Only One of the Five CheY Homologs in Vibrio cholerae Directly Switches Flagellar Rotation. J Bacteriol 187:84038410.

757 32. Teschler JK, Zamorano-Sánchez D, Utada AS, Warner CJA, Wong GCL, Linington RG, Yildiz FH. 2015. Living in the matrix: assembly and control of Vibrio cholerae biofilms. Nature reviews Microbiology 13:255-68.

33. Atuma C, Strugala V, Allen A, Holm L. 2001. The adherent gastrointestinal mucus gel layer: thickness and physical state in vivo. American Journal of Physiology-Gastrointestinal and Liver Physiology 280:G922-G929.

34. Johansson MEV, Sjövall H, Hansson GC. 2013. The gastrointestinal mucus system in health and

35. Podolsky DK, Isselbacher KJ. 1983. Composition of human colonic mucin. Selective alteration in inflammatory bowel disease. J Clin Invest 72:142-153.

36. Johansson MEV, Phillipson M, Petersson J, Velcich A, Holm L, Hansson GC. 2008. The inner of the two Muc2 mucin-dependent mucus layers in colon is devoid of bacteria. Proceedings of the National Academy of Sciences 105:15064-15069.

37. Freter R, O\&apos;Brien PC. 1981. Role of chemotaxis in the association of motile bacteria with intestinal mucosa: chemotactic responses of Vibrio cholerae and description of motile

773 38. Kočevar-Nared J, Kristl J, Šmid-Korbar J. 1997. Comparative rheological investigation of crude gastric mucin and natural gastric mucus. Biomaterials 18:677-681. 
40. Martinez Va, Schwarz-Linek J, Reufer M, Wilson LG, Morozov AN, Poon WCK. 2014. Flagellated bacterial motility in polymer solutions. Proc Natl Acad Sci U S A 111:17771-17776.

41. Lele PP, Hosu BG, Berg HC. 2013. Dynamics of mechanosensing in the bacterial flagellar motor. Proc Natl Acad Sci U S A 110:11839-11844.

42. Tipping MJ, Delalez NJ, Lim R, Berry RM, Armitage JP. 2013. Load-dependent assembly of the bacterial flagellar motor. mBio 4:e00551-13-e00551-13.

43. Lai SK, Wang Y-Y, Wirtz D, Hanes J. 2009. Micro- and macrorheology of mucus. Advanced drug delivery reviews 61:86-100.

44. Macierzanka A, Mackie AR, Krupa L. 2019. Permeability of the small intestinal mucus for physiologically relevant studies: Impact of mucus location and ex vivo treatment. Sci Rep 9:17516.

45. Georgiades P, Pudney PDA, Thornton DJ, Waigh TA. 2014. Particle tracking microrheology of purified gastrointestinal mucins. Biopolymers 101:366-77.

46. Theves M, Taktikos J, Zaburdaev V, Stark H, Beta C. 2013. A Bacterial Swimmer with Two Alternating Speeds of Propagation. Biophysj 105:1915-1924.

47. Cai Q, Li Z, Ouyang Q, Luo C, Gordon VD. 2016. Singly Flagellated Pseudomonas aeruginosa Chemotaxes Efficiently by Unbiased Motor Regulation. mBio 7:e00013.

48. Mukherjee T, Elmas M, Vo L, Alexiades V, Hong T, Alexandre G. 2019. Multiple CheY Homologs Control Swimming Reversals and Transient Pauses in Azospirillum brasilense. Biophys J $116: 1527-1537$.

49. Lapidus IR, Welch M, Eisenbach M. 1988. Pausing of flagellar rotation is a component of bacterial motility and chemotaxis. J Bacteriol 170:3627-3632.

50. Armitage JP, Macnab RM. 1987. Unidirectional, intermittent rotation of the flagellum of Rhodobacter sphaeroides. J Bacteriol 169:514-518. 
51. Qian C, Wong CC, Swarup S, Chiam K-H. 2013. Bacterial tethering analysis reveals a "run-reverseturn" mechanism for Pseudomonas species motility. Appl Environ Microbiol 79:4734 - 4743.

52. Eisenbach M, Wolf A, Welch M, Caplan SR, Lapidus IR, Macnab RM, Aloni H, Asher O. 1990. Pausing, switching and speed fluctuation of the bacterial flagellar motor and their relation to motility and chemotaxis. J Mol Biol 211:551-563.

53. Kojima M, Kubo R, Yakushi T, Homma M, Kawagishi I. 2007. The bidirectional polar and unidirectional lateral flagellar motors of Vibrio alginolyticus are controlled by a single CheY species. Mol Microbiol 64:57-67.

54. Sourjik V, Schmitt R. 1996. Different roles of CheY1 and CheY2 in the chemotaxis of Rhizobium meliloti. Mol Microbiol 22:427-436.

$812 \quad$ 55. Bible A, Russell MH, Alexandre G. 2012. The Azospirillum brasilense Che1 chemotaxis pathway controls swimming velocity, which affects transient cell-to-cell clumping. J Bacteriol 194:33433355.

56. Kudo S, Imai N, Nishitoba M, Sugiyama S, Magariyama Y. 2005. Asymmetric swimming pattern of Vibrio alginolyticuscells with single polar flagella. FEMS Microbiol Lett 242:221-225.

817 57. Grognot M, Taute KM. A multiscale 3D chemotaxis assay reveals bacterial navigation mechanisms. Communications Biology accepted

819 58. Morse M, Colin R, Wilson LG, Tang JX. 2016. The Aerotactic Response of Caulobacter crescentus. Biophys J 110:2076-2084.

821 59. Freter R, O'Brien PC. 1981. Role of chemotaxis in the association of motile bacteria with intestinal mucosa: chemotactic responses of Vibrio cholerae and description of motile nonchemotactic mutants. Infect Immun 34:215-221. 
824 60. Freter R, O\&apos;Brien PC. 1981. Role of chemotaxis in the association of motile bacteria with

825

826

827

828

829

830

831

832

833

834

835

836 intestinal mucosa: fitness and virulence of nonchemotactic Vibrio cholerae mutants in infant mice. Infect Immun 34:222-233.

61. Bubendorfer S, Koltai M, Rossmann F, Sourjik V, Thormann KM. 2014. Secondary bacterial flagellar system improves bacterial spreading by increasing the directional persistence of swimming. Proc Natl Acad Sci U S A 111:11485-11490.

62. Saragosti J, Silberzan P, Buguin A. 2012. Modeling E. coli tumbles by rotational diffusion. Implications for chemotaxis. PLoS One 7:e35412.

63. Son K, Menolascina F, Stocker R. 2016. Speed-dependent chemotactic precision in marine bacteria. Proceedings of the National Academy of Sciences 113:8624-8629.

64. Xie L, Lu C, Wu X-I. 2015. Marine Bacterial Chemoresponse to a Stepwise Chemoattractant Stimulus. Biophysj 108:766-774.

65. Moisi M, Jenul C, Butler SM, New A, Tutz S, Reidl J, Klose KE, Camilli A, Schild S. 2009. A Novel Regulatory Protein Involved in Motility of Vibrio cholerae. J Bacteriol 191:7027-7038.

66. Utada AS, Bennett RR, Fong JCN, Gibiansky ML, Yildiz FH, Golestanian R, Wong GCL. 2014. Vibrio cholerae use pili and flagella synergistically to effect motility switching and conditional surface attachment. Nature Communications 5:4913.

67. Hyakutake A, Homma M, Austin MJ, Boin MA, Hase CC, Kawagishi I. 2005. Only One of the Five CheY Homologs in Vibrio cholerae Directly Switches Flagellar Rotation. J Bacteriol 187:84038410.

68. Nishiyama S-i, Takahashi Y, Yamamoto K, Suzuki D, Itoh Y, Sumita K, Uchida Y, Homma M, Imada K, Kawagishi I. 2016. Identification of a Vibrio cholerae chemoreceptor that senses taurine and amino acids as attractants. Sci Rep 6:20866. 
847 69. Nhu NTQ, Wang HJ, Dufour YS. 2019. Acidic pH reduces Vibrio cholerae motility in mucus by weakening flagellar motor torque. bioRxiv 59:871475.

849 70. Boyd S, Parikh N, Chu E, Peleato B, Eckstein J. 2011. Distributed Optimization and Statistical Learning via the Alternating Direction Method of Multipliers. Foundations and $\operatorname{Trends}^{\circledR}$ in Machine Learning 3:1-122.

852 71. Frenkel EM, McDonald MJ, Dyken JDV, Kosheleva K, Lang GI, Desai MM. 2015. Crowded growth leads to the spontaneous evolution of semistable coexistence in laboratory yeast populations. Proceedings of the National Academy of Sciences 112:11306 - 11311.

855

72. Degnen ST, Newton A. 1972. Chromosome replication during development in Caulobacter crescentus. J Mol Biol 64:671-680. 

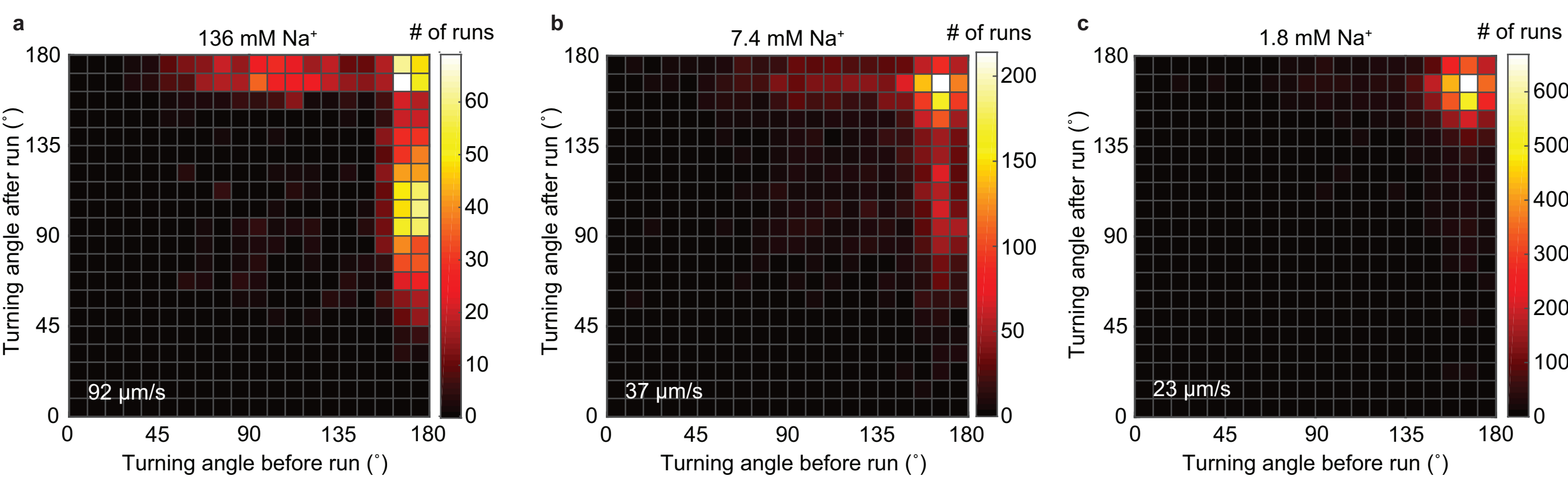


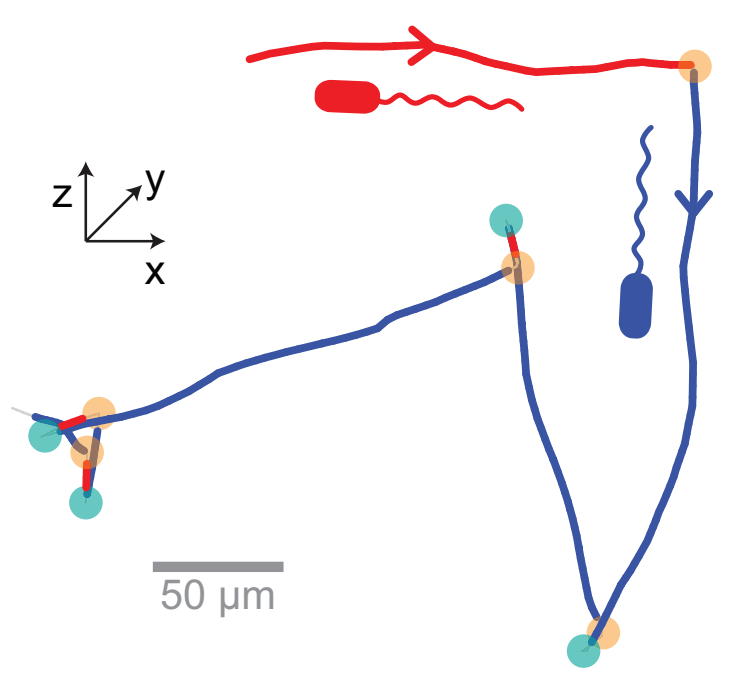

d

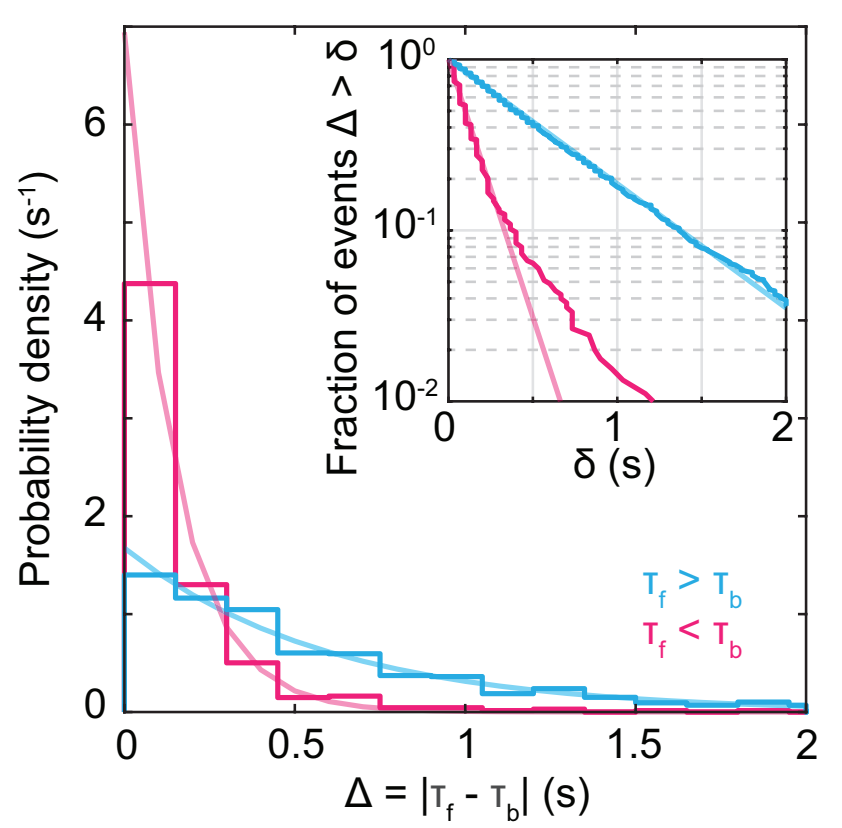

b

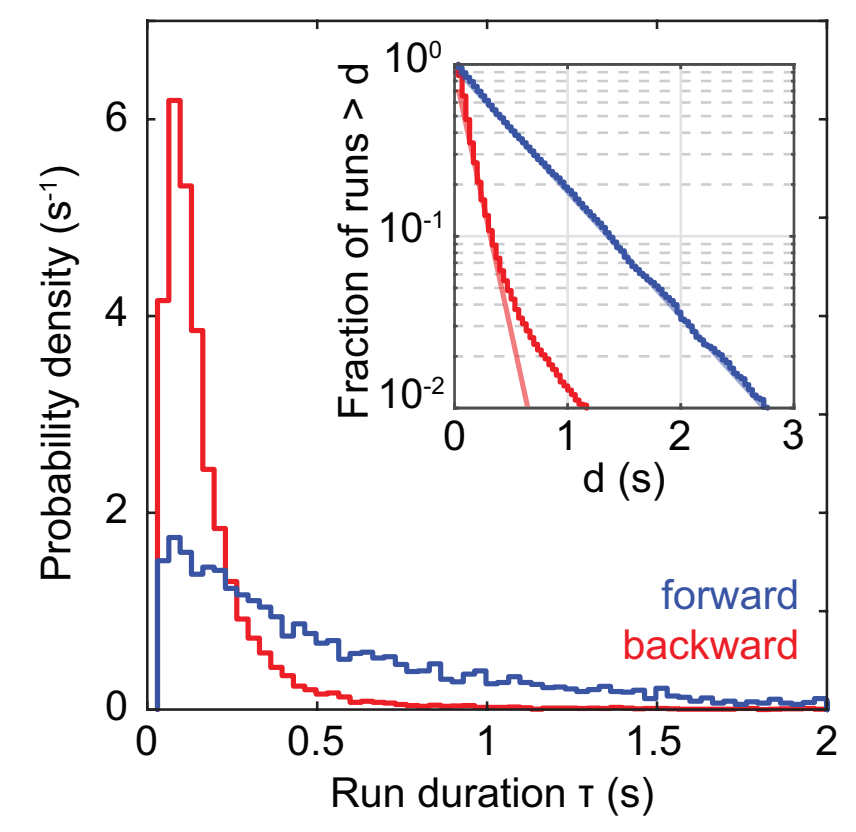

e

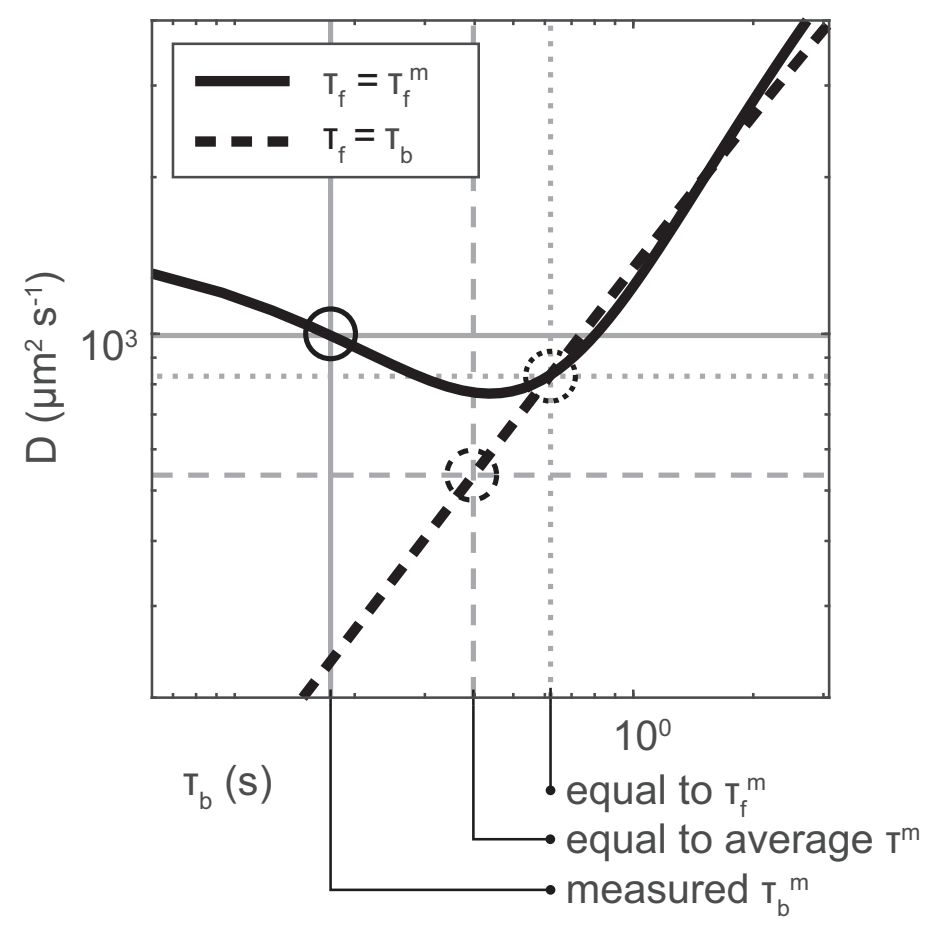

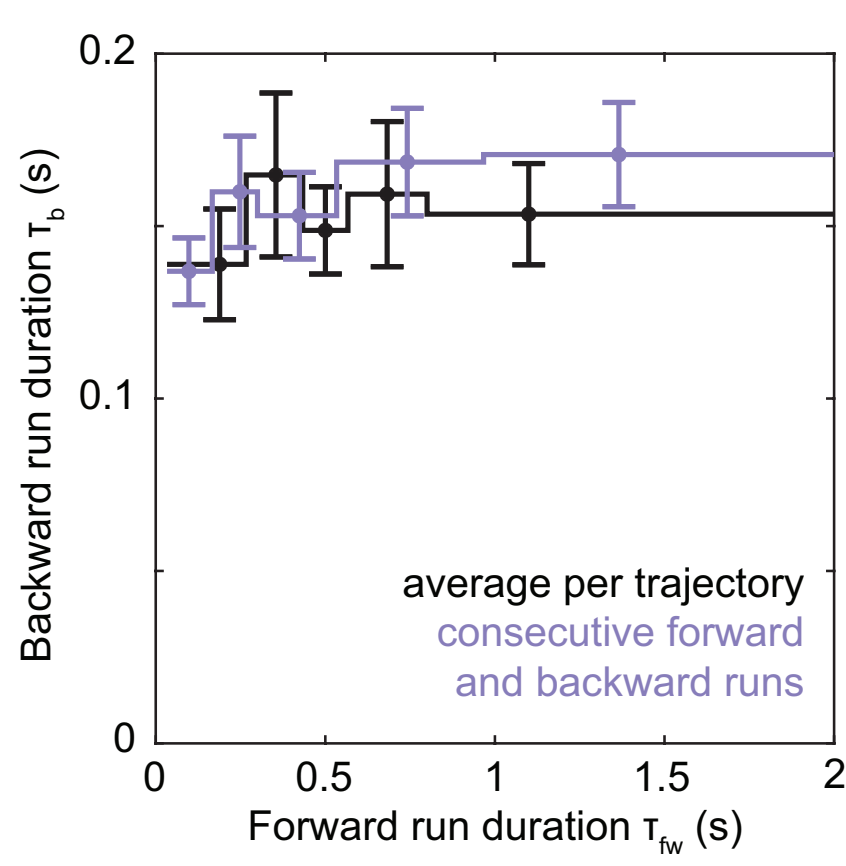



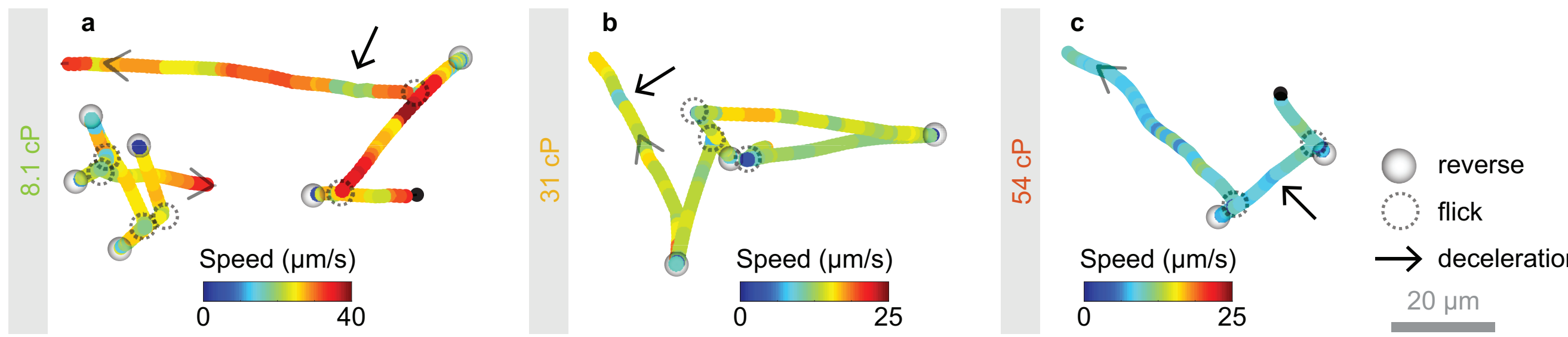

d
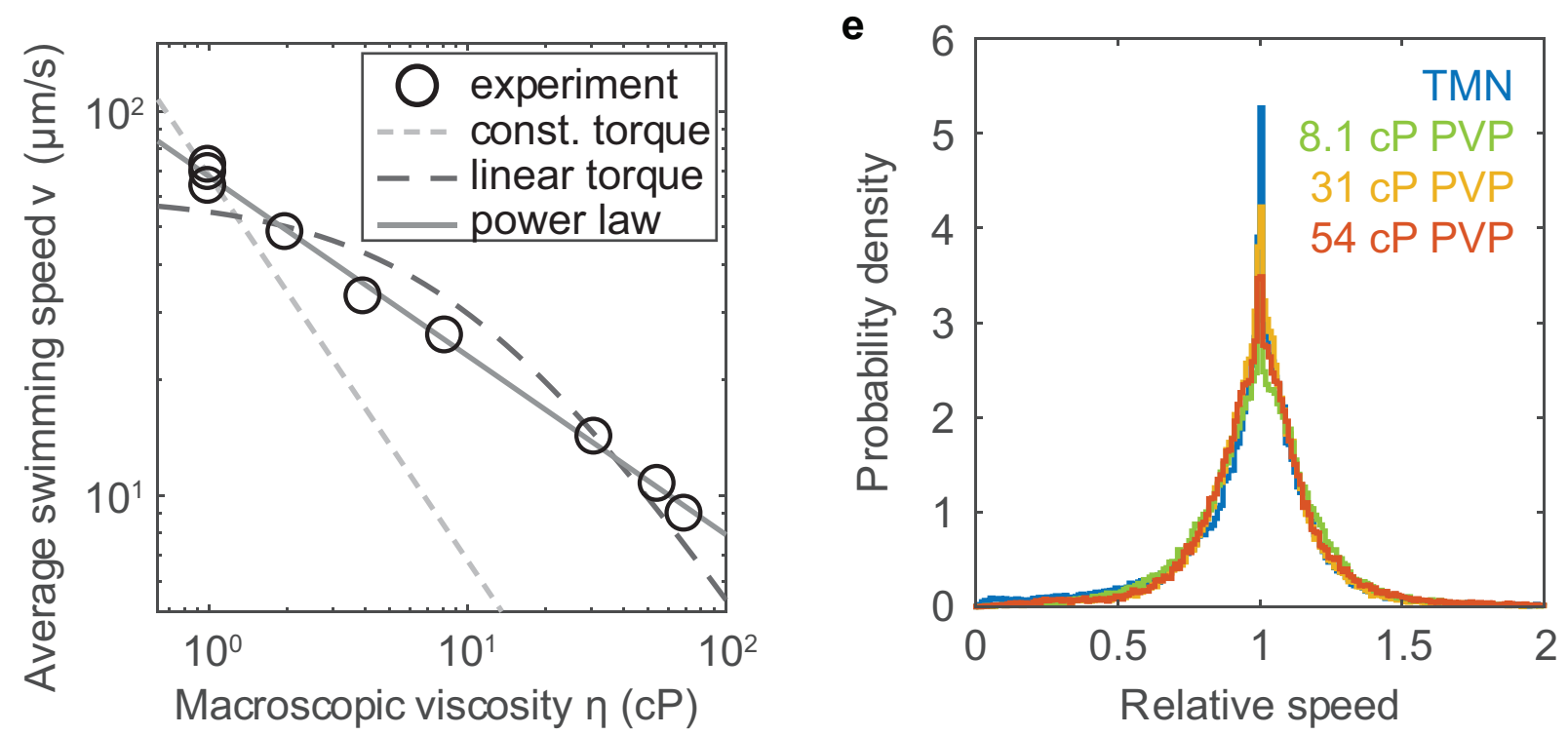